\title{
Intraparenchymal Hemorrhage of the Newborn
}

National Cancer Institute

\section{Source}

National Cancer Institute. Intraparenchymal Hemorrhage of the Newborn. NCI

Thesaurus. Code C117203.

Bleeding into the brain tissue of a newborn infant. 